\title{
Indicadores (Outcomes) Primários e Secundários em Ensaios Clínicos Oncológicos: Definição e Usos
}

\author{
Primary and Secondary Outcomes in Oncology Clinical Trials: Definitions \\ and Uses
}

António VAZ-CARNEIRO ${ }^{1}$, Ricardo da LUZ $^{1}$, Margarida BORGES ${ }^{1}$, João COSTA ${ }^{1}$

Acta Med Port 2014 Jul-Aug;27(4):498-502

\section{RESUMO}

Introdução: A prova de eficácia de uma intervenção terapêutica em oncologia consegue-se através de ensaios clínicos rigorosamente conduzidos. Um dos factores metodológicos mais importantes é a selecção de indicadores clínicos de eficácia (outcomes), necessários ao cálculo das chamadas medidas de associação que permitem a definição de eficácia terapêutica.

Material e Métodos: Foi feita uma revisão narrativa baseada em alguns dos documentos de agências reguladoras internacionais, assim como documentos de consenso entre as sociedades científicas oncológicas, procurando listar e avaliar criticamente cada um dos indicadores utilizados em ensaios clínicos oncológicos.

Resultados: Identificaram-se como indicadores mais importantes a sobrevivência global, a sobrevivência livre de progressão/sobrevivência livre de doença, a toxicidade/qualidade de vida e taxa objectiva de resposta tumoral.

Discussão: A selecção do outcome primário deve basear-se no conceito de eficácia terapêutica, mas também na toxicidade relativa da terapêutica experimental, na sobrevivência esperada após progressão da doença, na existência de fármacos alternativos já estudados com indicações idênticas e até a prevalência da patologia em causa.

Conclusão: A selecção de indicadores em ensaios clínicos oncológicos reveste-se de especial importância e a sua selecção deve ser bem fundamentada, dependendo da doença, dos doentes e do fármaco em estudo.

Palavras-chave: Ensaios Clínicos como Assunto; Neoplasias; Oncologia Médica; Resultado do Tratamento.

\section{ABSTRACT}

Introduction: The proof of efficacy from a therapeutic intervention in oncology must be defined through well conducted clinical trials. One of the most important methodological issue is the outcome selection needed to calculate measures of association allowing definition of clinical efficacy.

Material and Methods: We designed a narrative revision based on some of the international regulatory instructions from drug agencies, as well as consensus papers from scientific oncology societies, listing and critically assessing each outcome used in oncology clinical trials.

Results: We identified as being the most important outcomes in oncology trials the overall survival, the progression free survival/ disease-free survival, the toxicity, the quality of life/patient-reported outcomes and the objective response rate.

Discussion: The selection of the primary outcome must be based on therapeutic efficacy as well as toxicity, expected survival, alternative drug regimens and even disease prevalence.

Conclusion: The selection of efficacy outcomes for clinical trials in oncology is very important and its selection must be well justified, and depends on the type of disease, the patients and the drug being studied.

Keywords: Clinical Trials as Topic; Neoplasms; Medical Oncology; Treatment Outcome.

\section{INTRODUÇÃO}

O número de cancros diagnosticados a nível mundial apresenta uma incidência crescente e, para o seu tratamento, são necessárias provas científicas sólidas que determinem a eficácia e a segurança dos agentes estudados. ${ }^{1}$

A reclamação de eficácia de uma intervenção terapêutica - oncológica ou não - deve basear-se em estudos experimentais com metodologias rigorosas, permitindo o estabelecimento de uma associação causal entre exposição e resultado, definida por medidas de associação específicas. A metodologia cientificamente indicada para estes estudos é o ensaio clínico aleatorizado e controlado (Randomized Clinical Trial - RCT).

Ao longo das últimas décadas tem-se vindo a assistir a uma crescente complexidade metodológica dos RCTs, especialmente oncológicos. Apesar disso, as designações clássicas das diferentes fases dos RCTs têm-se mantido, muito por força de aspectos regulamentares: fase I (testa pela primeira vez o fármaco num pequeno grupo de pacientes para avaliação da dose mínima eficaz e da segurança); fase II (alarga o estudo a um grupo restrito de doentes com a patologia em causa para testar eficácia); fase III (estuda o perfil de segurança e eficácia em amostra alargada e mais representativa da população com a doença específica, avaliando a eficácia relativa ao tratamento padronizado ou ao placebo - este tipo de estudo serve habitualmente de base à aprovação pelas autoridades regulamentares da introdução do medicamento no mercado); fase IV (análise do perfil acima de tudo de segurança do fármaco que já está comercializado). A fase III é considerada a mais importante, porque define a dimensão da eficácia em comparação com placebo/substância activa/tratamento padronizado e porque pode definir um perfil de eficácia/segurança nos

1. Centro de Estudos de Medicina Baseada na Evidência. Faculdade de Medicina. Universidade de Lisboa. Lisboa. Portugal. Recebido: 20 de Fevereiro de 2014 - Aceite: 07 de Julho de 2014 | Copyright @ Ordem dos Médicos 2014 
doentes com indicação para fazerem a terapêutica com a molécula estudada, para além de ser a base para Autorização de Introdução no Mercado.

A investigação clínica em oncologia constitui presentemente uma das áreas mais importantes da actividade da indústria farmacêutica, demonstrada pelo facto de estarem a decorrer mais de 8000 RCTs estudando um elevado número de compostos. ${ }^{*}$ Adicionalmente, tem-se assistido na última década a uma alteração do paradigma da investigação clínica, em particular na oncologia, a qual passou a estar mais centrada em alvos terapêuticos específicos (muitas vezes determinados por características genéticas, e não tanto nas características fenotípicas dos doentes).

Neste artigo procuramos apresentar e discutir - de maneira necessariamente breve - um dos componentes metodológicos mais importantes nos RCTs oncológicos: a selecção e interpretação dos indicadores de eficácia e segurança. Estes indicadores referem-se exclusivamente aos estudos fase II/III, não sendo discutidos os de fase I.

Selecção e uso de indicadores (outcomes ou endpoints) em RCTs: diferenças entre resultados primários e secundários na interpretação de um RCT

Os objectivos dos RCTs são - como já foi dito - definir benefícios e riscos terapêuticos através de comparações de intervenções. Isto consegue-se com avaliações das respostas de indicadores/resultados (outcomes ou endpoints) através dos quais os grupos são comparados. A questão da selecção e interpretação destas variações passa portanto a ser um aspecto crucial no desenho e generalização dos resultados do RCT em causa.

Os outcomes podem classificar-se como primários e secundários (existem RCTs até com outcomes terciários, que não discutiremos). Os mais importantes são os primários, que são pré-especificados pela sua importância para os intervenientes envolvidos (doentes, médicos, investigadores, promotores, etc.), assim como para o cálculo da amostra. ${ }^{2,3}$ Os outros resultados de interesse são classificados como secundários e são variáveis de interesse para o estudo, habitualmente múltiplos, incluindo não só outras medidas de eficácia, como de segurança (este último aspecto é especialmente importante e os outcomes secundários devem integrar os efeitos não antecipados do tratamentos). ${ }^{4}$

Um outro aspecto a ter em conta é a utilização de outcomes de curta duração ou de conveniência, que podem não ter validade quando se tenta medir o impacto do tratamento. Estes 'marcadores substitutivos' (surrogate endpoints) podem não ser válidos como substitutos do outcome primário, por não possuírem as características prognósticas daquele. ${ }^{5}$ Por outras palavras, um indicador clínico é o que tem relevância clínica para o doente (por ex. mortalidade), sendo que o substitutivo obtém-se habitualmente de maneira mais fácil, muitas vezes por menor custo, ou de maneira menos invasiva, mas è crucial demonstrar o seu valor como factor prognóstico, ou seja, proceder à sua validação.

Pharmaceutical Executive, 8/2012

\section{Apresentação e discussão de indicadores em ensaios} clínicos oncológicos fase II/III**

A maior parte dos indicadores clínicos nos ensaios clínicos oncológicos fases II/III estão bem definidos e têm sido universalmente utilizados como marcadores de eficácia e segurança.

Como seria de esperar, a maior parte destes indicadores possuem relevância clínica (isto é, não são marcadores fisiológicos ou biológicos) e são algo diferentes no caso dos estudos de fase II (em que o indicador mais utilizado é a taxa de resposta tumoral) ou fase III (sobrevivência global).

$\mathrm{Na}$ Tabela 1 estão descritos os outcomes mais frequentemente utilizados (esta lista não é exaustiva) - sobrevivência global, sobrevivência livre de progressão/doença, toxicidade, qualidade de vida e taxa objectiva de resposta tumoral - sendo discutidos cada um, individualmente.

\section{Sobrevivência global (Overall Survival - OS)}

A Sobrevivência Global - definida como o tempo entre a inclusão/registo no estudo e a morte (por qualquer causa) - é o indicador mais importante em RCTs oncológicos.

Com efeito, a sua objectividade e relevância clínica são óbvias, a determinação é imediata e a precisão é sempre absoluta. Este indicador é imune ao chamado viés de investigador (em que este pode influenciar aquele). ${ }^{6}$

Sofre no entanto de alguns problemas, nomeadamente quando existe uma taxa elevada de doentes perdidos no seguimento (e portanto não analisados em termos de mortalidade) ou com mortalidade não-oncológica, podendo tornar o cálculo da mortalidade real e, por conseguinte da sobrevivência global, muito difícil.

Outro problema é a necessidade de, em cancros com baixa mortalidade ou respondedores ao tratamento de base, ser necessário uma amostra de grandes dimensões com seguimentos muito prolongados no tempo, para se poderem detectar diferenças estatisticamente significativas do tratamento (se, na altura da análise final, a maior parte dos doentes inda estão vivos, as estimativas de sobrevivência podem ser muito variáveis ou impossíveis de determinar).

Além disso, quando uma percentagem elevada de doentes do grupo de controlo acaba por receber o fármaco - ou durante o estudo (crossover), ou fora dele (por razões éticas) - verifica-se uma diluição da eficácia terapêutica entre os dois braços do estudo.

Finalmente, quando um novo fármaco é administrado em add-on, isto é, adicionado a uma terapêutica de base (já provadamente eficaz), a eficácia é mais dificilmente detectável, já que a sobrevivência é maior do que em doentes não tratados. ${ }^{7}$

Apesar destas limitações, e devido à sua extraordinária relevância clínica, a sobrevivência global deve ser o indicador preferido em ensaios clínicos fase III.

Um último alerta: na determinação do benefício-risco de um medicamento antineoplásico, a diferença de

** Este artigo refere-se a ensaios que testam medicamentos ou agentes biológicos, excluindo ensaios com imunoterapia. 
Tabela 1 - Outcomes principais em ensaios clínicos oncológicos

\begin{tabular}{|c|c|c|c|c|}
\hline INDICADOR & DEFINIÇÃO & TIPO DE ESTUDO & VANTAGENS & DESVANTAGENS \\
\hline $\begin{array}{l}\text { Sobrevivência } \\
\text { global (Overall } \\
\text { Survival -OS) }\end{array}$ & $\begin{array}{l}\text { O tempo entre a inclu- } \\
\text { são/registo no estudo e } \\
\text { a morte (por qualquer } \\
\text { causa) }\end{array}$ & Ensaios clínicos & $\begin{array}{l}\text { - Resultado muito relevante } \\
\text { - Fácil determinação } \\
\text { - Precisão } \\
\text { viés dos investigadores } \\
\text { - Baixo custo, não } \\
\text { necessitando de testes } \\
\text { específicos para sua detecção }\end{array}$ & $\begin{array}{l}\text { - Necessidade de estudos de } \\
\text { grandes dimensões } \\
\text { Inclusão de mortes não } \\
\text { oncológicas Influenciado por tratamento } \\
\text { cruzado e sequencial (pode ser } \\
\text { insensível a uma terapia inovadora } \\
\text { em crossover, por ex.) } \\
\text { - Abandono de doentes do RCT }\end{array}$ \\
\hline $\begin{array}{l}\text { Sobrevivência } \\
\text { livre de progres- } \\
\text { são (Progression } \\
\text { Free Survival } \\
\text { - PFS) na doen- } \\
\text { ça avançada I } \\
\text { Sobrevivência } \\
\text { Livre de Doença } \\
\text { (Disease-Free/ } \\
\text { Relapse Free } \\
\text { Survival - DFS) }\end{array}$ & $\begin{array}{l}\text { O intervalo entre o início } \\
\text { da terapêutica e o agra- } \\
\text { vamento da doença ou } \\
\text { a morte do doente por } \\
\text { qualquer causa }\end{array}$ & Ensaios clínicos & $\begin{array}{l}\text { - Menor dimensão amostral } \\
\text { - Menor tempo de } \\
\text { seguimento (followup) } \\
\text { - Medição de doença } \\
\text { estável } \\
\text { tratamentos subsequentes dos } \\
\text { tramcia de efeito domentes }\end{array}$ & $\begin{array}{l}\text { - O tempo de progressão não } \\
\text { pode ser conhecido com exactidão } \\
\text { - Necessidade de utilização de } \\
\text { imagiologia para a sua correcta } \\
\text { definição } \\
\text { - Habitual ausência de relação } \\
\text { entre os resultados imagiológicos e } \\
\text { o prognóstico do doente } \\
\text { - Ausência de validação em todos } \\
\text { os contextos } \\
\text { pariação na definição de estudo } \\
\text { para estudo }\end{array}$ \\
\hline
\end{tabular}

\begin{tabular}{|c|c|c|c|}
\hline Toxicidade & Taxa de efeitos adversos & $\begin{array}{l}\text { Ensaios clínicos } \\
\text { Farmacovigilância }\end{array}$ & $\begin{array}{l}\text { - Definição do perfil de } \\
\text { benefício/risco da terapêutica }\end{array}$ \\
\hline $\begin{array}{lr}\text { Qualidade } & \text { de } \\
\text { vida } & \text { (Quality } \\
\text { of Life }- & Q O L), \\
\text { Sintomas } & \text { Re- } \\
\text { portados } & \text { pelos } \\
\text { Doentes } & \end{array}$ & $\begin{array}{l}\text { Outcome auto-reportado } \\
\text { pelos doentes utilizando } \\
\text { escalas de bem-estar, } \\
\text { de presença de efeitos } \\
\text { adversos e de toxicidade } \\
\text { das terapêuticas }\end{array}$ & $\begin{array}{l}\text { Ensaios clínicos } \\
\text { Farmacovigilância }\end{array}$ & $\begin{array}{l}\text { Perspectiva directa dos } \\
\text { doentes sobre o benefícic } \\
\text { clínico }\end{array}$ \\
\hline $\begin{array}{l}\text { Taxa objectiva } \\
\text { de resposta tu- } \\
\text { moral (Objective }\end{array}$ & $\begin{array}{l}\text { Proporção de respon- } \\
\text { dedores (parciais ou } \\
\text { completos) da amostra }\end{array}$ & $\begin{array}{l}\text { RCTs de braço único ou } \\
\text { braços múltiplos } \\
\text { Ocultação mandatória }\end{array}$ & $\begin{array}{l}\text { Não é uma medida directa de } \\
\text { eficácia }\end{array}$ \\
\hline $\begin{array}{l}\text { Response Rate } \\
\text { - ORR) }\end{array}$ & global & & $\begin{array}{l}\text { Só um pequeno grupo de } \\
\text { doentes beneficia }\end{array}$ \\
\hline
\end{tabular}

- Por vezes os doentes não reportam com precisão os efeitos adversos

- Por vezes, difícil estabelecimento de causa-efeito

- $\quad$ Reporting por vezes incompleto - Pequenos sintomas e sinais de difícil avaliação

- Poucos instrumentos validados
- Definição precisa por vezes difícil - Impossibilidade de medição imagiológica de certos tumores - Intervalos de avaliação não normalizados eficácia - estatisticamente significativa - entre os dois grupos (experimental e controlo) deve ser de suficiente magnitude e acompanhada por uma toxicidade suficientemente aceitável para ser considerada clinicamente significativa.

\section{Sobrevivência Livre de Progressão (Progression Free Survival - PFS) na doença avançada ou Sobrevivência Livre de Doença (Disease-Free Survival - DFS) no con- texto adjuvante}

A Sobrevivência Livre de Progressão de Doença (Progression Free Survival - PFS) na doença avançada ou a Sobrevivência Livre de Doença (Disease-Free Survival DFS), no contexto adjuvante, são indicadores temporais que definem o intervalo entre o início da terapêutica e o agravamento da doença ou o intervalo entre a remissão/ tratamento e a recidiva, respectivamente.

Estes indicadores têm vantagens e problemas.

Uma das vantagens é o impacto de uma terapêutica oncológica poder conceptualmente ser medido pelo tempo que a doença volta a manifestar-se após finalização do trata- mento, daí este outcome ser importante para o doente. As PFS/DFS não são afectadas pelos tratamentos subsequentes aos RCTs, pelo que podem ser utilizados no contexto de tratamentos adicionais considerados benéficos.

Mas estes outcomes têm várias desvantagens: uma delas é que o tempo até progressão não pode ser conhecido com exactidão, já que apenas pode definir-se entre intervalos de avaliação clínica do doente (o que o faz depender do cronograma de consultas clínicas de seguimento). Outra desvantagem é a necessidade de utilização de imagiologia para a sua correcta definição, com todos os problemas daí decorrentes (falsos positivos e negativos, graus de precisão, etc.). Ainda um outro problema é a relação (ou ausência desta) entre os resultados imagiológicos e o prognóstico do doente (a diminuição do tumor pode não ter qualquer impacto na mortalidade).

A definição destes endpoints (PFS/DFS) varia em função da intenção terapêutica: por ex. num doente com metástases inoperáveis, o indicador de interesse é o crescimento tumoral ou a progressão da doença (neste caso o 
PFS refere-se ao tempo de agravamento da doença). Por outro lado, num doente com ressecção cirúrgica completa do tumor, o evento crucial é a recidiva da doença - neste caso o DFS mede o intervalo de tempo entre a cirurgia e a recorrência da doença.

Presentemente a PFS está sendo cada vez mais utilizada nas fases II e III dos RCTs na doença avançada.

A European Medicines Agency (EMA) recomenda que, se a PFS é seleccionado como outcome primário, então a sobrevivência global deve ser obrigatoriamente um outcome secundário e vice-versa (Oncology Working Party, Guideline on the evaluation of anticancer medicinal products in man EMA/CHMP/205/95/Rev.4, 13 December 2012). Nos casos de progressão lenta de um tumor, é defensável uma análise num ponto pré-definido, mas com a condição de todos os doentes estarem incluídos na amostra.

\section{Toxicidade}

A toxicidade dos tratamentos é naturalmente um dos indicadores mais importantes a ter em conta, já que ajuda a definir o perfil de eficácia/segurança de um fármaco individual.

Uma das classificações mais comumente utilizada é a Common Terminology Criteria for Adverse Events (CTCAE), do US Department of Health and Human Services do National Institutes of Health - National Cancer Institute dos EUA, actualmente na sua versão 4.0, o qual é baseado na incidência de efeitos adversos (EAs), definido como um sinal ou sintoma desfavoráveis e não intencional (que inclui um resultado laboratorial anormal), ou um sintoma ou patologia que está associada à terapêutica farmacológica ou intervencional com relação (ou não) com o tratamento ou intervenção. Este sistema define cinco graus de progressiva gravidade dos EAs ( 1 = leve, $5=$ morte).

A classificação dos EAs, por este sistema ou qualquer outro, deve ser rigorosa, para que a realidade da toxicidade seja devidamente captada no estudo.

\section{Qualidade de vida (Quality of Life - QoL) I Sintomas reportados pelos doentes (Patient-Reported Outcomes - PRO)}

A avaliação da qualidade de vida (Quality of Life - QoL) ou dos sintomas reportados pelos doentes (Patient-Reported Outcomes - PRO) constituem outcomes de grande importância nos RCTs oncológicos, particularmente quando a mortalidade (o benefício terapêutico fundamental) é de modesta dimensão.

Estes indicadores são habitualmente auto-reportados pelos doentes, são de difícil avaliação e algo dispendiosos de obter. ${ }^{7}$ Os aspectos mais importantes da QoL incluem o equilíbrio e autonomia físicos e mentais, assim como os sintomas provocados pela terapêutica (a informação espontânea do doente é aqui particularmente valiosa).

No passado, a toxicidade e a taxa de resposta foram utilizados como marcadores substitutivos de qualidade de vida, mas não é o caso presentemente: a toxicidade constitui certamente um dos aspectos da qualidade de vida (ex- ceptuando alterações bioquímicas assintomáticas), mas a taxa de resposta não, já que uma resposta terapêutica pode não se acompanhar de melhoria sintomática, por exemplo. ${ }^{6}$

Os índices de qualidade de vida incluem-se habitualmente como resultados secundários nos RCTs fase III.

\section{Taxa objectiva de resposta tumoral (Objective Respon- se Rate - ORR)}

Dado que o volume de um tumor é um factor prognóstico significativo, a diminuição (ou aumento) daquele tem sido utilizado como indicador de eficácia terapêutica, baseado em evidência que, para muitos tumores sólidos, agentes que induzam diminuição do volume tumoral apresentam uma probabilidade de melhoria da sobrevivência global ou outro indicador de tempo-para-um-evento..$^{7,8}$

A taxa objectiva de resposta tumoral define-se como a proporção de respondedores (parciais ou completos) da amostra global. Este indicador tem sido utilizado em muitos ensaios (especialmente ensaios de fase II), mas a sua utilização não recolhe unanimidade entre os investigadores: por exemplo, a EMA não o considera válido (Oncology Working Party, Guideline on the evaluation of anticancer medicinal products in man EMA/CHMP/205/95/Rev.4, 13 December 2012). Apesar disso, este outcome aparece utilizado em inúmeros ensaios clínicos oncológicos recentes. ${ }^{8-10}$

Este indicador apresenta problemas de definição, já que é calculado através de técnicas imagiológicas. No passado, uma taxa de resposta considerava-se eficaz quando detectava uma diminuição bidimensional do volume tumoral de $50 \%$ ou mais, reconfirmada às quatro semanas. ${ }^{7}$ Neste contexto, a progressão de doença era definida como um aumento de $25 \%$ da lesão, e a estabilização de doença como aqueles que não atingiam uma resposta parcial mas não atingiam critérios de progressão. ${ }^{6}$ É claro que o cálculo dos diâmetros lesionais de medição de resposta constitui um problema quando se utilizam imagens bidimensionais, com planos diversos, a partir de vários estádios de desenvolvimento tumoral, etc.

Desde o ano de 2000 que um grupo de investigadores oncológicos publicou normas de medição de eficácia imagiológica, recentemente actualizadas: o sistema "Response Evaluation Criteria in Solid Tumors" (RECIST). ${ }^{11}$ Este sistema é o utilizado mais frequentemente para medição da ORR.

Apesar da normalização de abordagens, mantêm-se alguns problemas na utilização deste outcome (mesmo que apenas em fases II): por exemplo, a impossibilidade de medição de certos tumores, a deterioração do doente sem provas imagiológicas de aumento tumoral, intervalos de avaliação imagiológica muito diferentes, técnicas imagiológicas díspares para avaliação do mesmo doente (TC seguida de RMN, por ex.), etc.

Em conclusão: com excepção de tumores muito sintomáticos (em que a sua diminuição resulta em melhoria clínica), a taxa de resposta tumoral não constitui um outcome apropriado para ensaios clínicos fase III devido a dificul- 
dades de medição, relevância clínica pouco clara e fraca discriminação prognóstica em termos de sobrevivência.

\section{DISCUSSÃO}

Os RCTs oncológicos fases II/III servem de base à pretensão de eficácia e segurança e, no seu desenho, são habitualmente ensaios de superioridade (isto é, que procuram demonstrar um benefício terapêutico acrescido). ${ }^{6}$

A selecção e interpretação de outcomes (primários ou secundários) revestem-se de grande importância, já que a reclamação de eficácia repousa nos seus resultados comparativos.

A selecção do outcome primário deve basear-se no conceito de eficácia terapêutica, mas também na toxicidade relativa da terapêutica experimental. $\mathrm{E}$ ainda em factores como sobrevivência esperada após progressão da doença, na existência de fármacos alternativos já estudados com indicações idênticas a até a prevalência da patologia em causa.

A relação entre indicadores de sobrevivência global (OS) e a sobrevivência livre de progressão (PFS) representa, segundo a EMA, uma abordagem metodológica estrita. Como já foi dito, esta agência recomenda que, se o PFS é seleccionado como outcome primário, então a OS deve ser obrigatoriamente um outcome secundário e vice-versa. Isto implica que, se a OS for classificada como um outcome

\section{REFERÊNCIAS}

1. Miller FG, Joffe S. Balancing access and evaluation in the approval of new cancer drugs. JAMA. 2011;305:2345-6.

2. Carneiro AV. Cálculo da dimensão da amostra em estudos clínicos: princípios metodológicos básicos. Rev Port Cardiol. 2003;22:1513-21.

3. Schulz KF, Grimes DA. Sample size calculations in randomised trials: mandatory and mystical. Lancet. 2005;365:24-5.

4. Moher D, Hopewell S, Schulz KF, Montori V, Gotzsche PC, Devereaux PJ, et al. CONSORT 2010 explanation and elaboration: updated guidelines for reporting parallel group randomised trials. BMJ. 2010;340:c869.

5. Kelsen DP. Surrogate endpoints in assessment of new drugs in colorectal cancer. Lancet. 2000;356:353-4.

6. Green S, Benedetti J, Smith A, Crowley J. Clinical trials in oncology. 3. Boca Raton: CRC Press; 2012.

7. Kelly WM, Halabi S. Oncology clinical trials. New York: DermosMedical; 2010.

8. Hensley ML, Blessing JA, Mannel R, Rose PG. Fixed-dose rate gem- secundário, então nas estimativas de tratamento deverá verificar-se uma tendência para a superioridade. Por outro lado, quando se verifica um efeito muito marcado na PFS, ou se verifica uma sobrevivência expectável após progressão, e/ou um perfil favorável de segurança, podem não ser necessárias estimativas precisas sobre a OS para aprovação.

\section{CONCLUSÃO}

Em resumo, a selecção de indicadores em ensaios clínicos oncológicos reveste-se de especial importância (por exemplo mortalidade global, qualidade de vida). Indicadores pouco precisos ou subjectivos podem induzir interpretações enviesadas ou inconsistentes da parte dos investigadores, o que pode pôr em causa os resultados dos estudos. Por outro lado, indicadores clínicos precisos mas pouco apropriados para a patologia sob estudo podem fornecer comparações que de facto são incorrectas.

\section{CONFLITO DE INTERESSES}

Os autores declaram não existir conflito de interesses.

\section{FONTES DE FINANCIAMENTO}

Os autores declaram que não existiu qualquer apoio financeiro.

citabine plus docetaxel as first-line therapy for metastatic uterine leiomyosarcoma: a Gynecologic Oncology Group phase II trial. Gynecol Oncol. 2008;109:329-34.

9. Yildiz I, Sen F, Basaran M, Ekenel M, Agaoglu F, Darendeliler E, et al. Response rates and adverse effects of continuous once-daily sunitinib in patients with advanced renal cell carcinoma: a single-center study in Turkey. Jpn J Clin Oncol. 2011;41:1380-7.

10. Kaseb AO, Shindoh J, Patt YZ, Roses RE, Zimmitti G, Lozano RD, et al Modified cisplatin/interferon alpha-2b/doxorubicin/5-fluorouracil (PIAF) chemotherapy in patients with no hepatitis or cirrhosis is associated with improved response rate, resectability, and survival of initially unresectable hepatocellular carcinoma. Cancer. 2013;119:3334-42.

11. Eisenhauer EA, Therasse P, Bogaerts J, Schwartz LH, Sargent D, Ford $\mathrm{R}$, et al. New response evaluation criteria in solid tumours: revised RECIST guideline (version 1.1). Eur J Cancer. 2009;45:228-47. 


\section{Indicadores (Outcomes) Primários e Secundários em Ensaios Clínicos Oncológicos: Definição e \\ Usos}

Acta Med Port 2014:27:498-502

Publicado pela Acta Médica Portuguesa, a Revista Científica da Ordem dos Médicos

Av. Almirante Gago Coutinho, 151

1749-084 Lisboa, Portugal.

Tel: +351218428215

E-mail: submissao@actamedicaportuguesa.com

www.actamedicaportuguesa.com

ISSN:0870-399X | e-ISSN: 1646-0758

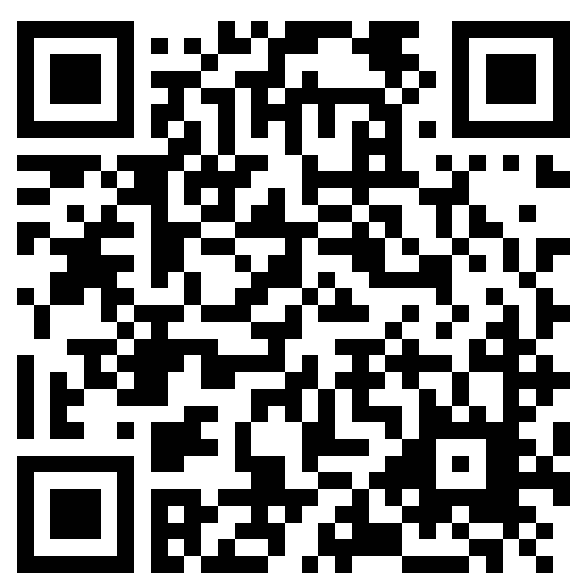

